\title{
DESIGN AND DEVELOPMENT OF JTAG USING RASPBERRY PI
}

\author{
Pilavendiran S.A \\ Department of ECE \\ Paavai Engineering College \\ Namakkal, Tamilnadu, India
}

\author{
Keshavarajan J.L \\ Department of ECE \\ Paavai Engineering College, \\ Namakkal, Tamilnadu, India
}

\author{
Dr.S.Vijayakumar \\ Department of ECE \\ Paavai Engineering College \\ Namakkal, Tamilnadu, India
}

\begin{abstract}
A motherboard contain a different microprocessor, microcontroller and power consumer. This are all working under a different BIOS program the individuals work with unique programming languages, so it is very complex to program each microprocessor, microcontroller, FPGA and etc., present in a mother board and cost to write the individual programs into concern chips is very high and the time to write BIOS program into each chips is long in existing system, Apart from long duration and high cost the debugging process takes place in individual chips thus to overcome all those drawbacks present in existing system. We use our project as a single board computer. This single board computer is combination of raspberry pi along with JTAG through LINUX operating system. The main goal of our project is to write BIOS program in various different chips present in motherboard through JTAG simultaneously and the programming cost is very less compared with current existing method and the total execution time is comparatively less than existing system.
\end{abstract}

Keywords- Raspberry Pi, JTAG (Joint Test Action Group), GUI, Actel FPGA, STM32 Microcontroller

\section{INTRODUCTION}

Nowadays many Industries manufacture millions of motherboards, in each target motherboard there are many types of microprocessor, microcontroller, FPGA are present in it and they have a separate BIOS (binary input and output) programs. BIOS means getting ready the chip to load or to execute a firmware without error. The chips are manufactured and loaded with this is why we goes for raspberry pi along with JTAG for their fast computation time and error check using boundary scan technique. BIOS programming software for each microprocessor, microcontroller and FPGA are unique and we need to buy those programming separately with the corresponding company thus the cost is very high, so we go for raspberry pi with JTAG interface to reduce the cost of buying programming software by storing the BIOS programming software in the internal memory of the raspberry pi. The JTAG cable is used to form a bridge between the raspberry pi and the target motherboard which consist of several microprocessor, microcontroller and FPGA. The transfer time by using JTAG debugger is less while compared to other programmers. The auto error detection and correction is achieved using JTAG cable simultaneously debugging is achieved by it. Raspberry pi performs faster and it act as single board computer, so raspberry pi is used to replace normal computers. Thus the industries save lots of money by replacing computers with raspberry pi board.

\section{PROPOSED AlgorithM}

\section{A. Raspberry pi -}

The Raspberry Pi is used around the world for it's portability and efficiency. The Raspberry $\mathrm{Pi}$ is used as single board computer to perform all the operations at a faster rate. The Raspberry Pi contains 20 GPIO pins and those pins allow JTAG to connect with Raspberry Pi. The Raspberry Pi is having inbuilt RAM(Random Access Memory) and ROM(Read Only Memory) .

The Raspberry Pi stored the BIOS programs in it's internal storage to access at any time. The Raspberry Pi performs every operations computer does for professional work purpose. The Raspberry Pi execute operations at a faster rate while compared to normal computers. The Raspberry Pi is a serial of small single-board computer.

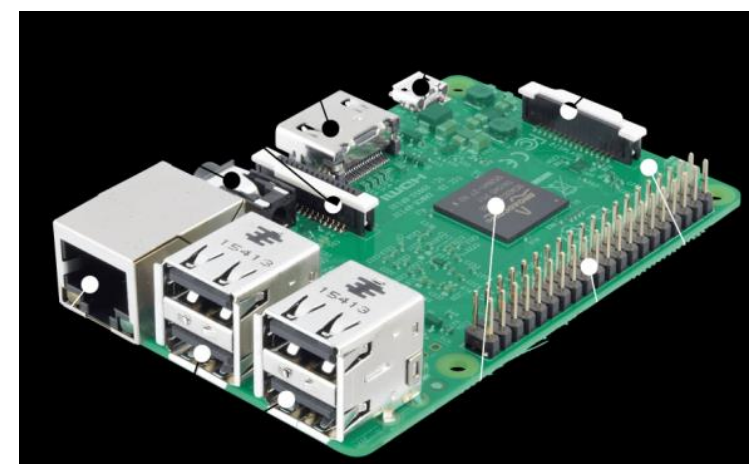

Fig. 1. Raspberry Pi board

Raspberry $\mathrm{Pi}$ is having 5V, 3A, full power delivery to USB devices. It is having a CPU of $1.5 \mathrm{GHz}, 64 / 32$-bit quad- 


\section{International Journal of Engineering Applied Sciences and Technology, 2020 \\ Vol. 4, Issue 10, ISSN No. 2455-2143, Pages 75-79 \\ Published Online February 2020 in IJEAST (http://www.ijeast.com)}

core ARM cortex-A72. It has SoC of BCM2837 and Central Processing Unit of Quad Cortex A53 @1.2GHz.

Table 1: Raspberry Pi GPIO configuration for JTAG

\begin{tabular}{|l|l|l|l|}
\hline Register & Pin & Configuration & $\begin{array}{l}\text { JTAG } \\
\text { signal }\end{array}$ \\
\hline GPFSEL0 & GPIO4 & ALT5 & TDI \\
\hline GPFSEL2 & GPIO22 & ALT4 & TRST \\
\hline GPFSEL2 & GPIO24 & ALT4 & TDO \\
\hline GPFSEL2 & GPIO25 & ALT4 & TCK \\
\hline GPFSEL2 & GPIO27 & ALT4 & TMS \\
\hline
\end{tabular}

Raspberry Pi is having instruction set of ARMv8-A.It has GPU of $400 \mathrm{MHz}$ Video Core IV and the RAM (random access memory) of 1GB SD RAM. It has Micro-SD slot present in it with Ethernet 10/100. The wireless is containing 802.11n/Bluetooth 4.0 and Video output of HDMI/Composite. Audio output of HDMI/Headphone.

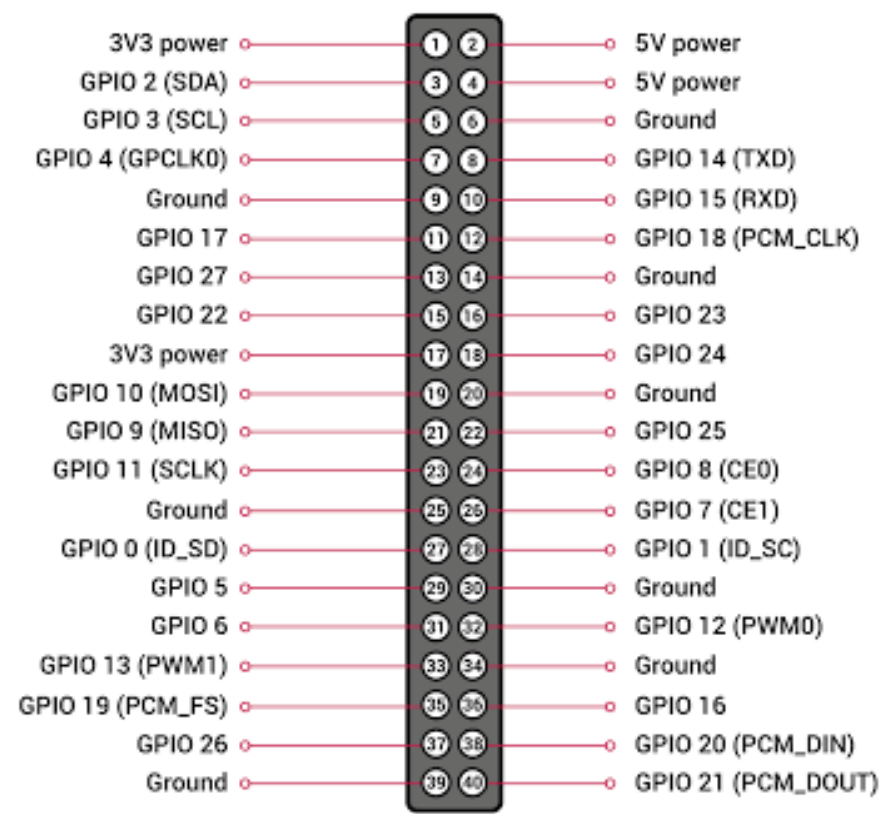

Fig. 2. GPIO-Raspberry Pi

\section{B. JTAG -}

JTAG is abbreviated as Joint Test Action Group. It's an industry standard for verifying designs and testing printed circuit boards after manufacture. It's a serial communication interface for low overhead access without requiring direct external access to the system address and data buses. It's able to access to all chips on a target circuit mother board. follows

JTAG is having four primary pins they are given as

- TDI

- TDO
- $\quad$ TMS

- TCK

- $\quad$ TRST

TDI is abbreviated as Test data input TDO is abbreviated as Test data output TMS is abbreviated as Test mode select TCK is abbreviated as Test clock TRST is abbreviated as Test reset

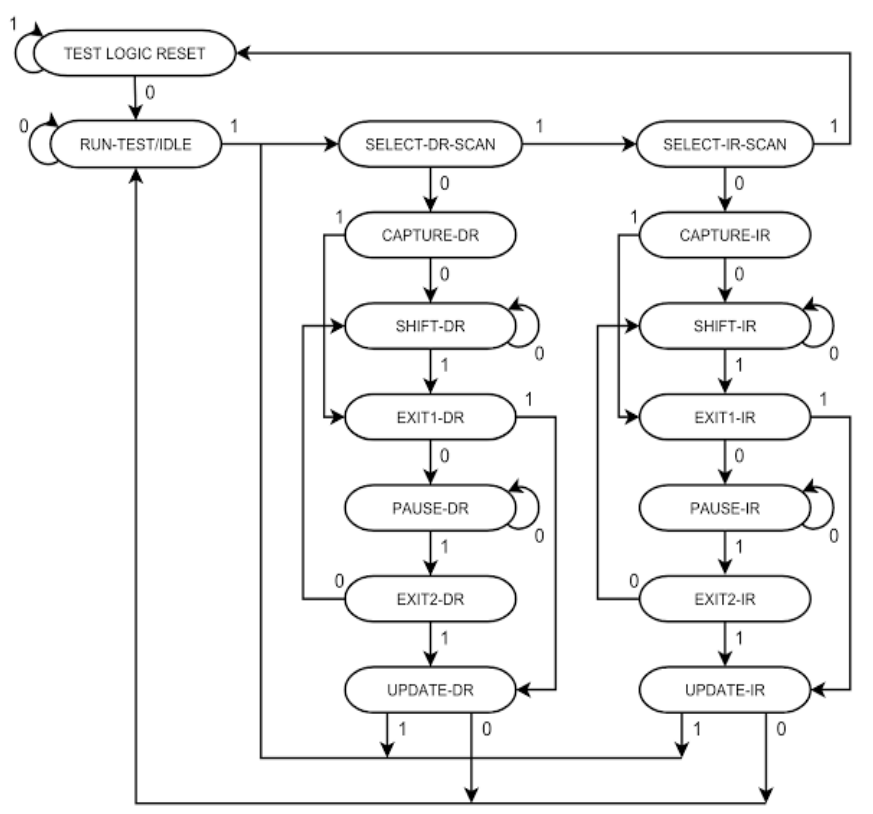

Fig. 3. JTAG TAP controller state diagram

\section{GUI -}

The GUI stands for graphical interface may be a interface thst allows user to interact with electronic devices through graphical icons and audio. A GUI uses a mixture of technologies and devices to supply a platform that users can interact with, for tasks of gathering and producing information.

In our project GUI is employed to make a decision that which program must install during which chip. Thus programming of multiple chips is formed possible without error thanks to presence of GUI (graphical user interface). there'll be no trouble in wrongly programming multiple chip because GUI decides program to be installed in each chip set present during a target motherboard.

GUI is used to create the graphical user interface to execute the BIOS program into chip. The execution is done in LINUX operating system present within the Raspberry Pi. Through JTAG the BIOS program is transferred into chip and the desire of choosing BIOS program to write in chip is done in GUI. 


\section{International Journal of Engineering Applied Sciences and Technology, 2020 \\ Vol. 4, Issue 10, ISSN No. 2455-2143, Pages 75-79 \\ Published Online February 2020 in IJEAST (http://www.ijeast.com)}

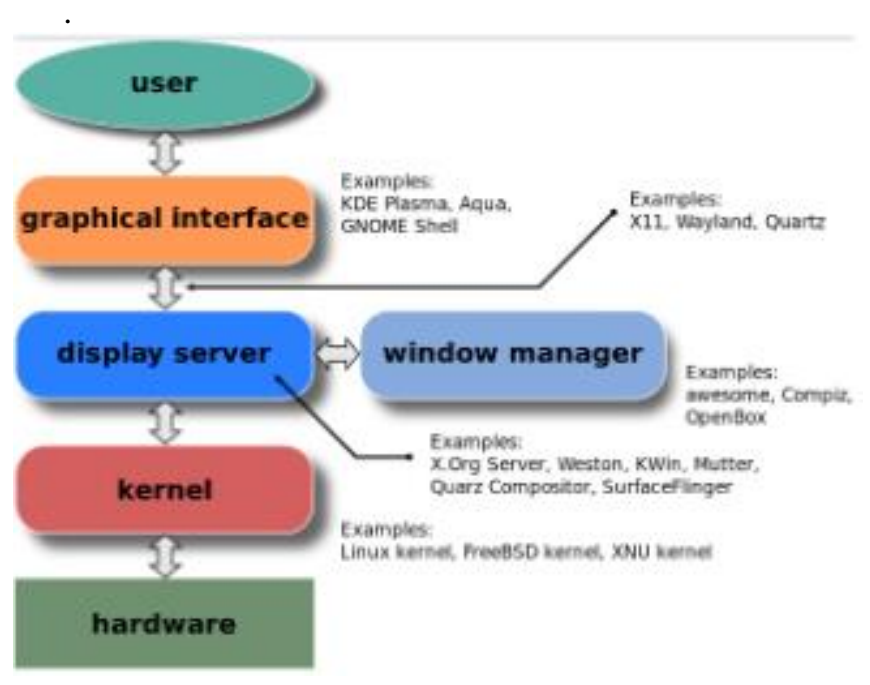

Fig. 4. Layers of graphical user interface

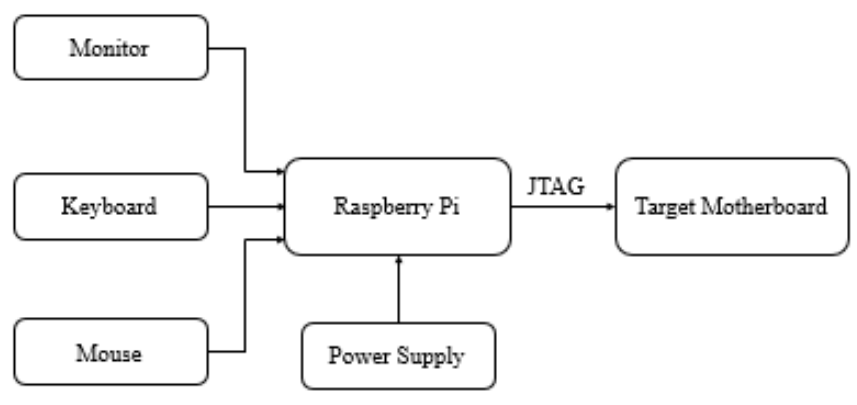

Fig. 5. Proposed Block Diagram

\section{Linux kernal -}

This section describes the way to enable JTAG on RPi for debugging Linux kernel. Linux may be a default choice as a general purpose OS for RPi [4]. Kernel debugging isn't a trivial task, but doing it remotely simplifies the approach tons. By default, JTAG isn't enabled within the kernel, so special steps are needed before the kernel are often debugged over JTAG.

The kernel compilation for RPi follows similar steps as for x 86 processors, for instance. Like bare metal programs, the compiler should be configured to create programs for ARM processors.

The kernel expects to be loaded at the address 0x8000. But usually the kernel image is placed on the booting partition during a compressed form as (zImage) alongside decompress or. A decompress or is ignorant to where it's loaded to, and it's smart enough to uncompressed the kernel and put it to the right memory address. This is often important because when building a picture for the RPi which will enable JTAG before booting the kernel, the particular zImage are going to be shifted in memory to introduce the JTAG enabling code ahead of it.

The RPi community provides the script to make the image kernel.img which will be put to the cardboard from a newly compiled Linux kernel. The script puts alittle bootloader at the address $0 x 0$, the kernel arguments at the address 0x100 and zImage at 0x8000. The bootloader and therefore the arguments are provided as text files with hexadecimal strings most likely converted from binary files.

Following an equivalent idea, it's possible to convert the bare metal JTAG enabling program into a document with the specified hexadecimal strings and put it at $0 \times 8000$, in order that JTAG gets enabled first. During this case, zImage is appended then code.

In order to be ready to debug the kernel booting process from the very beginning, the JTAG enabling software can enter an infinite loop, in order that the kernel booting should be started manually: by opening an OpenOCD session, halting the execution, and jumping to the address, where zImage ended up being loaded to.

So, the kernel are often debugged over JTAG from the very beginning of the booting process. But during its execution some kernel drivers might override the GPIO pins used for JTAG for his or her purposes. All GPIO JTAG pins have some alternative configurations for other functions. Possible overriding candidates are I2 C (Inter-Integrated Circuit) and SPI (Serial Peripheral Interface) drivers. Neither I2 C, nor SPI signal pins overlap with those of JTAG. Quite that, both $\mathrm{I} 2 \mathrm{C}$ and SPI drivers are disabled within the kernel by default.

\section{E. Open OCD -}

The OpenOCD is an open-source tool for debugging embedded systems. It runs on the host machine as a server, allowing connections over Telnet or from GDB (The GNU Project Debugger), so as to debug the target systems, connected to the host machine through a JTAG adapter.

Since there are many variant of the adapters, also as many JTAG implementations, OpenOCD should be configured to figure with a specific adapter and therefore the board.

\section{EXPERIMENT AND RESULT}

The Raspberry Pi contains the BIOS programs for each microprocessor, microcontroller, FPGA present in a target motherboard. By using GUI (graphical user interface) the BIOS programs are instructed to install into concern chips it can be either microprocessor, microcontroller, FPGA. Thus JTAG transfer the data into respective chip sets present in the target motherboard simultaneously.

Therefore, the debugging is achieved through JTAG debugger and programming of multiple chips is made possible in a less amount of time, minimum power consumption and 


\section{International Journal of Engineering Applied Sciences and Technology, 2020 \\ Vol. 4, Issue 10, ISSN No. 2455-2143, Pages 75-79 \\ Published Online February 2020 in IJEAST (http://www.ijeast.com)}

low cost through JTAG using Raspberry Pi in Linux operating system.

\section{ANALYSIS TABULATION}

Table 2: SuperPro 610P

\begin{tabular}{|c|c|}
\hline Device name & SuperPro 610P \\
\hline $\begin{array}{l}\text { Device } \\
\text { supported }\end{array}$ & $\begin{array}{l}\text { EPROM, Paged EPROM, Parallel and serial } \\
\text { EEPROM, BPROM , NVRAM, SPLD, } \\
\text { CPLD, EPLD, Firmware HUB, } \\
\text { Microcontroller, MCU }\end{array}$ \\
\hline PC interface & USB 2.0 \\
\hline Power supply & $\begin{array}{l}\text { AC adapter: Input AC } 100 \mathrm{~V}-240 \mathrm{~V} \\
\text { Output: } 12 \mathrm{~V} / 1.5 \mathrm{~A}\end{array}$ \\
\hline Speed & 14 Seconds \\
\hline PIN & 48-pin \\
\hline $\begin{array}{l}\text { Programming } \\
\text { Testing } \\
\text { features }\end{array}$ & TTL/CMOS logic ICs and memories \\
\hline Compatible & Windows XP/ Vista/ 7/ 8/ 10 (32/64 bit) \\
\hline Cost & 35,289 Rupees \\
\hline
\end{tabular}

Table 3: Raspberry Pi with JTAG

\begin{tabular}{|c|c|}
\hline Connector Type & JTAG \\
\hline Device supported & All embedded systems \\
\hline PC interface & Bluetooth 5.0, USB Type C \\
\hline Operating temperature & 0 -50 degrees $\mathrm{C}$ ambient \\
\hline Speed & $\begin{array}{l}10 \mathrm{x} \text { faster than existing } \\
\text { system }\end{array}$ \\
\hline PIN & 40-GPIO pin \\
\hline RAM & $4 \mathrm{~GB}$ \\
\hline Processor & $\begin{array}{l}\text { Quad core Cortex-A72 } \\
\text { (ARM v8 )64-bit SoC @ } \\
1.5 \mathrm{GHZ}\end{array}$ \\
\hline Compatible & Linux \\
\hline Cost & 5000 Rupees \\
\hline
\end{tabular}

Table 4: Reading and Writing times of some chips

\begin{tabular}{|l|l|l|l|}
\hline Chip type & Writing & Reading & Verify \\
\hline EN25T80 & $5.4 \mathrm{~S}$ & $1.2 \mathrm{~S}$ & $1.2 \mathrm{~S}$ \\
\hline SST25VF010A & $4.2 \mathrm{~S}$ & $0.6 \mathrm{~S}$ & $0.6 \mathrm{~S}$ \\
\hline
\end{tabular}

\begin{tabular}{|l|l|l|l|}
\hline W25X16 & $7.6 \mathrm{~S}$ & $2.3 \mathrm{~S}$ & $2.3 \mathrm{~S}$ \\
\hline KH25L8005 & $4.3 \mathrm{~S}$ & $1.3 \mathrm{~S}$ & $1.3 \mathrm{~S}$ \\
\hline AT24C512 & $5.8 \mathrm{~S}$ & $3.6 \mathrm{~S}$ & $3.6 \mathrm{~S}$ \\
\hline AT24C256 & $3.0 \mathrm{~S}$ & $1.8 \mathrm{~S}$ & $1.8 \mathrm{~S}$ \\
\hline $\begin{array}{l}\text { ST25P16 } \\
\text { [SOP8] }\end{array}$ & $7.3 \mathrm{~S}$ & $2.3 \mathrm{~S}$ & $2.3 \mathrm{~S}$ \\
\hline
\end{tabular}

\section{CONCLUSION}

The debugging and programming of several microprocessors, microcontrollers and FPGA present in target motherboard is processed simultaneously with less consumption of time. The cost of programming is reduced several times compared to existing method due to pre-stored program present in internal storage of raspberry pi and auto error correction is achieved through JTAG debugger.

\section{REFERENCE}

1. Alexander P J, N. Radhakrishnan "Remote Lab Implementation on an Embedded Web Server" 2015 International Conference on Circuit, Power and Computing Technologies [ICCPCT].

2. Anzhelika Parkhomenko, Aleksandr Sokolyanskii, Olga Gladkova, Sergey Kurson "Investigation of Remote Lab Design Technologies" MEMSTECH 2015, 2-6 September, 2015, Polyana-Svalyava (Zakarpattya), UKRAINE.

3. Arkhomenko Anzhelika, Gladkova Olga, Eugene Ivanov, Aleksandr Sokolyanskii, Sergey KursonP "Development and Application of Remote Laboratory for Embedded Systems Design” 978-1-4799-7839-7/15/\$31.00 (C2015 IEEE.

4. Ivan Ganchev, Zhanlin Ji, M'airt'1n O'Droma “A Generic IoT Architecture for Smart Cities" ISSC 2014 / CIICT 2014, Limerick, June 26-27.

5. Martin Kalu'z, L'ubos` $\mathrm{C}^{\smile}$ irka and Miroslav Fikar "Simplifying the Implementation of Remote Laboratories in Educational Environments Using Industrial Hardware" 2013 International Conference on Process Control (PC) June 18-21, 2013, Štrbské Pleso, Slovakia.

6. Shuang Wang; Huiyang Zhao, "Design and implementation of remote virtual network lab," Systems and Informatics (ICSAI), 2012 International Conference on, vol., no., pp.1005, 1007, 19-20 May 2012.

7. Vini Madan , S.R.N Reddy "GSM-Bluetooth based Remote Monitoring and Control System with Automatic Light Controller" International Journal of Computer Applications (0975 - 8887) Volume 46- No.1, May 2012. 
8. Xu Wei, "The Research of Embedded Database of Mass Storage Technology", microcomputer Information, (in Chinese). (24):119-120 2008.

9. Yeoh, K.J.M.; Hwee Ling Wong, "Web-based remote navigational robot for multiclass human-robot interaction," Sustainable Utilization and Development in Engineering and Technology (STUDENT), 2012.

10. Zhang Quan-gui, "Embedded Internet and the application in the monitoring and control system, Information Technology", vol.28no. 4, pp52-54, 2004. 